\title{
Bone Metastases in Patients with Neuroendocrine Neoplasm: Frequency and Clinical, Therapeutic, and Prognostic Relevance
}

\author{
Michael Scharf ${ }^{\mathrm{a}}$ Vanessa Petry ${ }^{\mathrm{a}}$ Hanna Daniel $^{\mathrm{b}}$ Anja Rinke $^{\mathrm{a}}$ \\ Thomas Matthias Gress ${ }^{\text {a }}$ \\ Departments of a Gastroenterology, Endocrinology, Metabolism and Infectiology and ${ }^{\mathrm{b}}$ Medical Biometry and \\ Epidemiology, Philipps University, Marburg, Germany
}

\section{Keywords}

Bone metastases · Neuroendocrine neoplasia - Prognosis .

Clinical relevance $\cdot$ Therapy

\begin{abstract}
Background: The incidence and prevalence of neuroendocrine neoplasms (NEN) are rising. In view of continuously improving imaging techniques, more than half of the patients present with distant metastases at initial diagnosis. An advanced disease stage negatively correlates with the 5-year survival rate. In stage IV disease, bone metastases (BM) are frequent, yet knowledge concerning their clinical or prognostic relevance is rare. This study presents a single-center experience on the frequency and management of BM in patients with gastroenteropancreatic NEN and lung carcinoids. Methods: Between 2000 and June 2015, 327 of 677 patients treated in the European Neuroendocrine Tumor Society (ENETS) center in Marburg (Germany) presented with distant metastases (48.3\%), including 85 patients (12.6\%) with BM. Data of both groups were analyzed using descriptive statistics. Overall survival was assessed by Kaplan-Meier curves
\end{abstract}

Anja Rinke and Thomas M. Gress share last authorship.

\section{KARGER}

() 2017 S. Karger AG, Basel

E-Mail karger@karger.com

www.karger.com/nen and compared by log-rank test. Results: Median age in the BM group was 54.9 years, the small intestine and the pancreas being the most common primaries. $83.5 \%$ of the tumors were well and moderately differentiated (G1/G2). Nearly half of the patients with BM were symptomatic and suffered either from pain (42.4\%) or had fractures (11.7\%). Bisphosphonates were employed in almost two-thirds of the patients, radiation therapy in $25.9 \%$. Overall survival was significantly inferior in patients with BM than in those with other distant metastases ( $p=0.01 ; 49.0$ vs. 100.8 months). Conclusion: BM appear to have a significant clinical and prognostic impact. Further studies are needed to evaluate therapeutic approaches directed to the treatment of BM in particular for asymptomatic patients. @ 2017 S. Karger AG, Basel

\section{Introduction}

Neuroendocrineneoplasms(NEN) of thegastroenteropancreatic (GEP) system are rare tumors with a low, yet steadily increasing incidence and an already high prevalence [1]. While all GEP-NEN are summarized under a common nomenclature and are graded based on the WHO classification of 2010 into neuroendocrine tumor

Anja Rinke

Department of Gastroenterology and Endocrinology, Philipps University Baldinger Strasse

DE-35043 Marburg (Germany)

E-Mail sprengea@ staff.uni-marburg.de 
(NET) G1 (Ki-67 <2\%), NET G2 (Ki-67 3-20\%) or neuroendocrine carcinoma G3 (Ki-67 >20\%), they are heterogeneous in terms of clinical manifestation, prognosis and metastatic capacity [2]. Throughout the past years, our understanding of the tumor biology and pathophysiology of GEP-NEN and lung carcinoids has improved. Apart from identifying new gene mutations such as those of the DAXX/ATRX complex in pancreatic NETs, a deeper understanding of somatostatin receptor signaling as well as new insights into the mTOR pathway and tumor angiogenesis have led to the development of additional systemic therapies. Nowadays, among others, molecularly targeted treatments such as everolimus and sunitinib for pancreatic NET patients are readily used in addition to somatostatin analogs and the prognosis of patients with advanced disease has thereby significantly improved $[3,4]$.

Nevertheless, the best treatment option depends on the individual situation of the patient and may include surgical procedures (tumor debulking, resection of the primary), medical treatments as well as interventional procedures. In the presence of other distant metastases such as bone metastases (BM), instead of liver-directed therapies such as transarterial chemoembolization and selective internal radiation therapy, systemic therapies are preferred $[5,6]$. At the time of initial diagnosis, between 44 and $73 \%$ of patients at specialized NET centers in Europe already present with distant metastases reflecting a stage IV disease [5, 7-9]. Advanced disease stage per se is a significant predictor of prognosis [5]. BM occur in approximately $6-12 \%$ of the patients with NEN, are usually accompanied by other distant metastases $[9,10]$ and together with liver metastases are the major cause of death [11]. While the mechanisms responsible for the development of BM have not been fully understood, there is evidence that the RANK/RANKL/OPG pathway plays a major role and that the presence of alterations on this pathways may predict the development of BM in NEN patients [12].

Patients with $\mathrm{BM}$ may present with pain as a main symptom, but may also develop pathological fractures, neurological deficits or symptoms due to hypercalcemia, which have a huge impact on quality of life and treatment of the patients [6]. More frequently, BM are asymptomatic and are incidentally detected during staging or restaging of NETs in which case their relevance, both in terms of prognostic value and choice of treatment, remains uncertain. Since 2 studies reported that the incidence of BM in patients with NET was 10 times higher in a postmortem analysis as compared to the clinical evalu- ation (42 vs. $4 \%$ ) $[13,14]$, it appears that the incidence of $\mathrm{BM}$ is underestimated. Due to new imaging technologies with ${ }^{68} \mathrm{Ga}$-labeled tracers, the detection rate of $\mathrm{BM}$ has already increased and will continue to do so. A recent review comparing the 3 most commonly used tracers (DOTATATE, DOTATOC, and DOTANOC) reported a sensitivity well above $90 \%$ and a specificity close to $100 \%$ for BM [15]. Overall, at present, we have little information on the clinical course and treatment of GEP-NET patients with $\mathrm{BM}$, even though $\mathrm{BM}$ are more frequently diagnosed and need to be taken into account to design an individual treatment strategy. The aim of our single-center retrospective study is to evaluate BM in NEN patients in terms of frequency, clinical manifestation, impact on overall survival (OS) as well as treatment strategies.

\section{Patients and Methods}

\section{Patients}

All patients treated in the European Neuroendocrine Tumor Society (ENETS) center in Marburg, Germany, between January 2000 and June 2015 were evaluated for inclusion into this retrospective study. Patients had to be 18 years of age or older and had previously given their consent to be registered in our NET database and to the use of their medical history for research studies. Furthermore, only patients with GEP-NEN, unknown or pulmonary primaries were included. All NEN were staged according to the ENETS staging system for GEP-NEN [2] and pulmonary carcinoids [16]. Among the patients in our general database, we identified a subgroup of patients with distant metastases and therefore stage IV disease, which comprises patients with BM detected either at initial presentation or during follow-up. Diagnosis of BM had to be confirmed either by imaging such as X-ray, MRI scan, CT scan, bone scintigraphy, somatostatin receptor scintigraphy (Octreoscan $\left.{ }^{\circledR}\right)$, DOTATOC-PET-CT, and FDG-PET-CT or by histopathology in biopsy material. For both groups, patients with distant metastases as well as patients with BM, data were retrieved from our NET database and cross-checked with the medical records available either in print or in our hospital information system (Orbis ${ }^{\circledR}$, Agfa HealthCare). Information regarding grading and proliferation rate of the tumor (Ki-67 index value), location of the primary, sites of metastases, functional activity, and type of hormone production was collected. Furthermore, relevant time points such as the first date of NEN diagnosis, the date of first diagnosis of distant metastases and, if applicable, the date of death or at least the date the patient was last seen were determined. BM were classified according to their location into 6 different regions (upper and lower extremities, ribs, spine, pelvis, and head) and patients with $\mathrm{BM}$ were divided into 2 groups based on the period between initial diagnosis of the NEN and the diagnosis of BM: (i) synchronous BM that were diagnosed within 2 months after the first diagnosis of NEN and (ii) metachronous BM that were diagnosed more than 2 months after the diagnosis of NEN. In the peer group, metastases were classified according to their location (liver, lung, peritoneum, and others). Symptoms and complications re- 
lated to the BM such as pain, imminent and/or manifest pathological fractures, neurological symptoms, and hypercalcemia were recorded. Additionally, treatment or change of treatment after the first diagnosis of skeletal lesions was analyzed for all patients with $\mathrm{BM}$, primarily regarding the usage of bisphosphonates, RANK ligand inhibitors, palliative radiation or surgery when complications occurred. If contradictory or missing information were noticed, we attempted to contact the patient directly and when this was not possible, the patient was excluded from the study.

\section{Statistical Analysis}

All data were analyzed using the R software version 3.2.1 (June $18,2015)$. Descriptive statistics were employed to evaluate the frequency of BM, associated symptoms, interventions and general patient information. Data are reported either as real figures, percentages or medians with their ranges.

Median OS was calculated from the time point of the first diagnosis of distant metastases (stage IV disease) to the date of death or the date of the last presentation at our center. To calculate the survival of patients with BM, we used the onset of BM/first diagnosis of BM as the starting time point. For the comparison with the stage IV patient without BM, the time point of onset of stage IV seemed to be the most appropriate starting time point to calculate survival times. Patients and physicians are regularly contacted to update the follow-up information in our database. Kaplan-Meier curves were used to assess the median OS in the various subgroups and log-rank tests were employed to compare OS and survival after the diagnosis of stage IV in patients with and without BM. In our study design, calculation of progression-free survival times was not feasible. This was in part due to the retrospective nature of our study design and partly due to our function as ENETS center and national referral center for neuroendocrine neoplasias. Many of our patients live long distances away from our center and only visit our center's in- or outpatient facilities in longer intervals or when progress occurs and a change of treatment is required. While our center guides the overall treatment, basic care and follow-up is mostly done by physicians or oncologists close to the patients' home. Since BM were not assessed by RECIST criteria, a reliable distinction between progressive BM versus progression of other distant metastases as the reason for progressive disease was not possible either. In this overall setting, the precise time point of progression cannot be determined with enough accuracy to calculate progression-free survival times. Prognostic significance of BM was also evaluated using a multivariate Cox regression model that comprised resection of the primary tumor and the age at initial diagnosis as time-dependent covariate, and was stratified for grading and primary tumor location. The proportional hazard assumption of the Cox model was assessed by inspecting plots of Schoenfeld residuals. Results were considered statistically significant for $p$ values $<0.05$ and two-sided $p$ values are reported.

\section{Results}

\section{General Characteristics}

Based on the local database of the ENETS center of the University of Marburg, Germany, 677 patients treated at the hospital between January 1, 2000 and June 1, 2015 were identified for inclusion in this retrospective study. 327 of these 677 patients presented with distant metastases and were therefore classified as stage IV disease (48.3\%), while $85(12.6 \%)$ were reported with BM, either at the initial diagnosis of the NET $(n=30 ; 35.3 \%)$ or diagnosed during follow-up ( $n=55 ; 64.7 \%) .25$ patients (3.7\%) were excluded following our study protocol, mainly due to missing data. The median age in the BM group was 54.9 years at initial diagnosis and 57.3 years at the time of diagnosis of BM. Median age of patients with synchronous BM and other synchronous metastases was nearly identical (56.7 vs. 56.8 years). The most common location of the primary tumor in our study cohort was the small intestine $(n=32 ; 37.6 \%)$ closely followed by the pancreas $(n=26 ; 30.6 \%) .80$ out of 85 patients $(94.1 \%)$ also had liver metastases, while 1 patient solely presented with BM. Furthermore, BM were divided into 6 different body regions. The vertebra $(n=65 ; 76.5 \%)$ was the most frequent site of metastases followed by the pelvic region $(n=41 ; 48.2 \%)$ and the ribs $(n=27 ; 31.8 \%)$. None of the included patients had a multiple endocrine neoplasia syndrome. 37 patients were functionally active $(43.5 \%)$ with carcinoid syndrome ( $n=33,38.8 \%$ of all patients) being the most prevalent hormone syndrome, followed by insulinoma and Zollinger-Ellison syndrome with 3.5\% ( $n=$ $3)$ and $4.7 \%(n=4)$, respectively. Well and moderately differentiated NEN (G1/G2) made up 83.5\% $(n=71)$ of all patients with BM, while $14(16.5 \%)$ had a poorly differentiated tumor (G3). Due to the characteristic long life expectancy of patients with NEN, various different therapies are usually employed throughout the course of the disease. In our study cohort, for instance, only 15 patients (17.6\%) had no previous treatment, while over $60 \%(n=$ 54) had 2 or more therapies at the time of the first detection of BM. One patient had 6 different previous treatments. The most common therapies were somatostatin analog $(n=32 ; 37.6 \%)$, chemotherapy $(n=20 ; 23.5 \%)$, and transarterial chemoembolization ( $n=18 ; 21.2 \%)$, while only roughly $10 \%$ of all patients had a previous targeted therapy with everolimus $(n=8 ; 9.4 \%)$ or sunitinib $(n=2 ; 2.4 \%)$.

The composition of our peer group of 242 patients with distant metastases other than BM was comparable with $55.0 \%$ male $(n=133)$ and $45.0 \%$ female patients $(n=109)$. Median age was slightly older with 57.5 years. 87.0\% had a G1/G2 and $13.0 \%$ a G3 NEN. The proportion of small intestine NEN was higher $(n=111 ; 45.9 \%)$, while the number of pancreatic primaries $(n=66 ; 27.3 \%)$ was similar. Further characteristics of both groups are shown in Table 1.
Scharf/Petry/Daniel/Rinke/Gress 
Table 1. Characteristics of patients with bone metastases $(\mathrm{BM})(n=85)$ and other distant metastases $(n=242)$

\begin{tabular}{|c|c|c|c|c|c|}
\hline \multirow[t]{2}{*}{ Characteristics } & \multicolumn{2}{|c|}{ Bone metastases } & \multicolumn{2}{|c|}{$\begin{array}{l}\text { Distant metastases other than } \\
\text { bone }\end{array}$} & \multirow[t]{2}{*}{$\begin{array}{l}p \\
\text { values }\end{array}$} \\
\hline & $\begin{array}{l}\text { patients, } \\
n(\%)\end{array}$ & $\begin{array}{l}\text { age, years } \\
\text { (range) }\end{array}$ & $\begin{array}{l}\text { patients, } \\
n(\%)\end{array}$ & $\begin{array}{l}\text { age, years } \\
\text { (range) }\end{array}$ & \\
\hline Gender & & & & & 0.57 \\
\hline Male & $43(50.6)$ & & $133(55.0)$ & & \\
\hline Female & $42(49.4)$ & & $109(45.0)$ & & \\
\hline Median age at first diagnosis & & $54.9(23.5-79.6)$ & & $57.5(17.9-84.8)$ & 0.10 \\
\hline Median age at diagnosis of BM or distant metastases & & $57.3(24.4-79.7)$ & & $58.0(17.9-84.8)$ & 0.09 \\
\hline Primary tumor localization & & & & & 0.04 \\
\hline Small intestines & $32(37.6)$ & & $111(45.9)$ & & \\
\hline Pancreas & $26(30.6)$ & & $66(27.3)$ & & \\
\hline Rectum & $7(8.2)$ & & $6(2.5)$ & & \\
\hline Lung & $7(8.2)$ & & $7(2.9)$ & & \\
\hline Unknown & $11(12.9)$ & & $30(12.4)$ & & \\
\hline Duodenum & $1(1.2)$ & & $6(2.5)$ & & \\
\hline Appendix & $1(1.2)$ & & $3(1.2)$ & & \\
\hline Histology & & & & & 0.54 \\
\hline G1/G2 or differentiated & $71(83.5)$ & & $208(87.0)$ & & \\
\hline G3 or poorly differentiated & $14(16.5)$ & & $31(13.0)$ & & \\
\hline Median Ki-67 value, \% (range) & $5(1-90)$ & & $2.5(1-90)$ & & \\
\hline Functionality & & & & & 0.57 \\
\hline Functioning & $37(43.5)$ & & $91(39.2)$ & & \\
\hline Nonfunctioning & $48(56.5)$ & & $141(60.8)$ & & \\
\hline \multicolumn{6}{|l|}{ First diagnosis of $\mathrm{BM}$} \\
\hline Synchronous metastases & $30(35.3)$ & & & & \\
\hline Metachronous metastases & $55(64.7)$ & & & & \\
\hline
\end{tabular}

Ki-67 values were available for 80 and 204 patients, respectively.

\section{Detection of BM, Frequency and Treatment of Complications}

Approximately one-third of all patients with skeletal lesions reported pain associated with BM at initial diagnosis $(n=24 ; 28.2 \%)$ and another 12 patients $(14.1 \%)$ developed bone pain during ongoing treatment. Overall pain remained the principal symptom in $42.4 \%(n=36)$. Fractures occurred in merely $7.1 \%(n=6)$ at the time of diagnosis and increased to $11.8 \%(n=10)$ in the further course of the disease, while another 3.5\% $(n=3)$ had lesions that were classified as at risk for fracture. Only 1 patient $(1.2 \%)$ had an unstable vertebral fracture that was treated surgically. Only 4 out of 85 patients (4.7\%) with $\mathrm{BM}$ reported neurological deficits, all of them due to compression of the spinal cord by vertebral metastases and consecutive fractures of the vertebral bodies. Hypercalcemia was reported in only 1 case (1.2\%).

Diagnosis of BM led to a specific change or supplementation of the ongoing treatment in 71.8\% $(n=61)$. The most common treatments used were bisphospho- nates $(n=55 ; 64.7 \%)$, followed by palliative radiation therapy $(n=22 ; 25.9 \%)$. Only 3 patients received surgical therapy (3.5\%), and 6 patients received denosumab (7.1\%). Apart from BM-directed treatment, the most commonly used medical treatments after diagnosis were peptide receptor radionuclide therapy (PRRT) $(n=25$; $29.4 \%)$ and chemotherapy $(n=17 ; 20.0 \%) .69$ out of 85 patients $(81 \%)$ received BM-directed treatments. Although there probably is a bias in the selection of patients for treatment, patients with bone-specific treatments had a nonsignificant trend for a longer median OS compared to those without specific treatments ( 49.0 vs. 37.4 months, $p=0.39$ ).

The initial diagnosis of $\mathrm{BM}$ in the 85 patients analyzed in this study was predominantly done by MRI ( $n=36$; $42.4 \%$ ), whereas functional imaging either with somatostatin receptor scintigraphy or DOTATOC-PET-CT was responsible for the initial diagnosis of BM in 36.5\% $(n=31)$ of the patients (Table 2). In 6 of 85 patients (7.1\%), the initial diagnosis was determined by more than

Neuroendocrinology 2018;106:30-37 DOI: $10.1159 / 000457954$ 


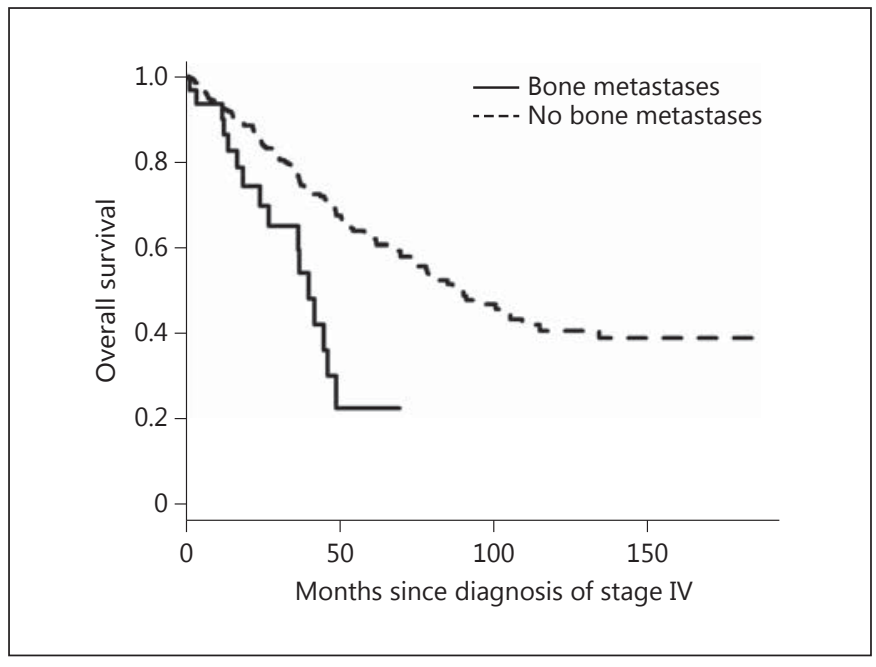

Fig. 1. Kaplan-Meier plot of overall survival measured from time of initial diagnosis of stage IV in patients with synchronous bone metastases or other metastases. Median overall survival in patients with synchronous bone metastases was significantly shorter than in patients with other metastases defining stage IV: 40.1 versus 90.1 months, $p<0.01$.

one imaging technique simultaneously. Since the introduction of Ga-DOTATOC-PET-CT imaging in our center, the frequency of BM detected in our NEN patients has increased. While 16 out of 84 patients with distant metastases (19.0\%) were diagnosed with BM in 2012 and 2013 when exclusively somatostatin receptor scintigraphy was used, 32 out of 98 patients (32.7\%) showed skeletal lesions in 2014/2015 when exclusively Ga-DOTATOC-PET-CT was used. This corresponds to a relative rise of the frequency of BM in patients with distant metastases of $72 \%$, which we attribute to the improved performance of GaDOTATOC-PET-CT for the detection of BM.

\section{Prognostic Relevance of BM}

Median survival from the time of initial diagnosis of $\mathrm{BM}$ was 36.7 months. To study the prognostic relevance of skeletal lesions we compared the median OS of patients with BM starting from the initial time of diagnosis of distant metastases $(n=85)$ with the median OS in our larger population $(n=242)$ with stage IV disease without BM.

Median OS in patients with synchronous BM was significantly shorter than in patients with stage IV disease caused by distant metastases other than BM: 40.1 versus 90.1 months, $p<0.01$ (Fig. 1). In the same way, the median OS in stage IV patients with BM diagnosed at any time during follow-up (calculated from the time of initial

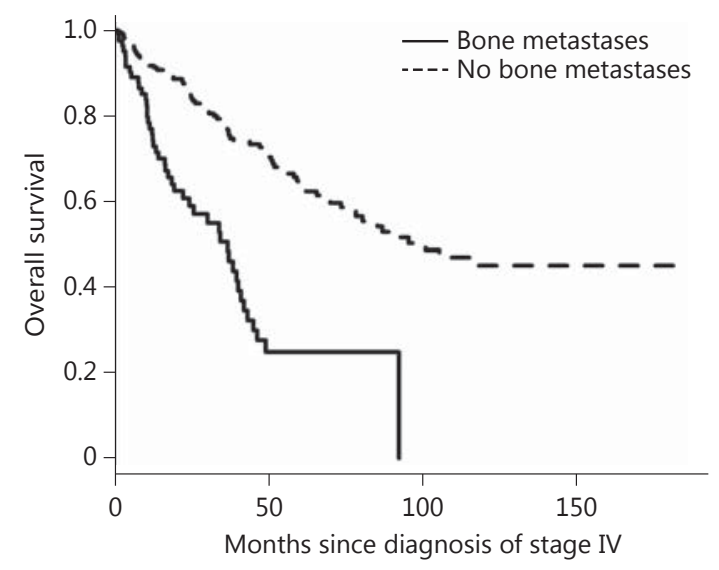

Fig. 2. Kaplan-Meier plot of overall survival of stage IV patients with bone metastases and stage IV patients without bone metastases calculated from the point of diagnosis of distant metastases. The median overall survival in stage IV patients with bone metastases diagnosed during follow-up was significantly inferior compared to that in patients with distant metastases other than bone metastases ( $p=0.01 ; 49.0$ vs. 100.8 months).

Table 2. Method used for initial detection of bone metastases (BM)

\begin{tabular}{lc}
\hline $\begin{array}{l}\text { Method used for initial } \\
\text { detection of BM }\end{array}$ & Patients, $n(\%)$ \\
\hline SMS & $13(15.3)$ \\
Bone scintigraphy & $5(5.9)$ \\
CT & $12(14.1)$ \\
MRI & $36(42.4)$ \\
DOTATOC-PET-CT & $18(21.2)$ \\
X-ray & $1(1.2)$ \\
Other PET-CTs & $4(4.7)$ \\
Unknown & $3(3.5)$ \\
Biopsy & $1(1.2)$ \\
\hline
\end{tabular}

In some cases multiple methods were employed. SMS, somatostatin receptor scintigraphy; CT, computed tomography; MRI, magnetic resonance imaging; PET, positron emission tomography.

diagnosis of distant metastases) was significantly worse than in patients with distant metastases without $\mathrm{BM}(p=$ $0.01 ; 49.0$ vs. 100.8 months). The related Kaplan-Meier curves are shown in Figure 2.

In a multivariate regression analysis using the timedependent covariate age at initial diagnosis and primary tumor resection stratified for grading and primary tumor 
Table 3. Multivariate analysis using the time-dependent covariate age at initial diagnosis and primary tumor resection stratified for grading and primary tumor location

\begin{tabular}{|c|c|c|c|}
\hline Covariate & $\operatorname{HR}(95 \% \mathrm{CI})$ & $\begin{array}{l}p \\
\text { value }\end{array}$ & $\begin{array}{l}\text { Global } \\
p \text { value }\end{array}$ \\
\hline Age & $1.03(1.02,1.05)$ & & $<0.01$ \\
\hline Grading & & & $<0.01$ \\
\hline $\mathrm{G} 1 / \mathrm{G} 2$ & reference & & \\
\hline G3 & $6.7(4,11.22)$ & & \\
\hline Primary tumor & & & 0.13 \\
\hline Small intestines & reference & & \\
\hline \multicolumn{4}{|c|}{ Other than small intestines } \\
\hline or pancreas & $0.3(0.09,0.98)$ & 0.05 & \\
\hline Pancreas & $0.32(0.09,1.07)$ & 0.06 & \\
\hline \multicolumn{2}{|l|}{ Primary tumor operation } & & $<0.01$ \\
\hline Yes & reference & & \\
\hline No & $2(1.35,2.95)$ & & \\
\hline \multicolumn{3}{|l|}{ Bone metastases } & $<0.01$ \\
\hline Other patients & reference & & \\
\hline \multicolumn{4}{|l|}{ Synchronous bone } \\
\hline metastases & $2.5(1.43,4.38)$ & & \\
\hline
\end{tabular}

location, the diagnosis of BM remained a statistically significant variate. In this analysis, skeletal lesions detected in patients at the time of the diagnosis of advanced disease had a hazard ratio of 2.5 (95\% CI: 1.43-4.38). Furthermore, a primary tumor left in place and older age had a significant negative impact on median OS with a hazard ratio of 2.0 and 1.03 per year, respectively, even though the difference for older age was neglectable. All results of our multivariate analysis are shown in Table 3.

We also compared the OS starting from the time of the detection of distant metastases in patients with symptomatic BM $(n=37,43.5 \%)$ with the OS in patients with asymptomatic BM $(n=48,56.5 \%)$. Median OS in BM patients without symptoms was significantly longer (79.0 vs. 42.0 months; $p=0.03$ ).

\section{Discussion}

\section{Rising Incidence of BM and Preferential Primary Site}

Even though a guideline for the management of BM has been published by the ENETS [6], relevant data are still rare. Due to the improved performance of new imaging technology, in particular ${ }^{68} \mathrm{Ga}$-DOTATOC-PET-CT, the diagnosis of skeletal lesions is steadily increasing $[17,18]$. This observation is supported by our own study showing a $72 \%$ relative increase in the proportion of patients with BM at first presentation when comparing 2 periods of time, one

Bone Metastases in NEN Patients before and one after the introduction of ${ }^{68} \mathrm{Ga}$-DOTATOCPET-CT at our center. In our database, BM were reported in 85 patients which represented $12.6 \%$ of all registered cases and $26.0 \%$ of all patients with distant metastases. This is slightly above the rate of detection previously found in the literature and comparable to the data reported in 2 recent studies from the United States $[19,20]$. As has been suggested before, the true incidence of BM may even be higher [6] since, as in our center, the use of DOTATOCPET-CT for routine staging of NEN patients has only recently become more common and in parallel the quality of other cross-sectional imaging is rapidly improving.

Whether the primary site of the tumor has an influence on the risk of BM has been a matter of debate. While some authors found no preferential primary site, Bhosale et al. [21], who studied whether the metastases detected by imaging allowed to predict the site of the primary tumor, reported a clear correlation between BM and primary tumors of either the lung or the large bowel [21, 22]. Interestingly, while the most common sites of the primary tumor in our study and in the entire cohort registered in our database were the small intestine and the pancreas, half of our patients with a rectal or pulmonary primary and distant metastases also had BM (rectal + BM $n=7,53.8 \%$; pulmonary $+\mathrm{BM} n=7,50.0 \%$, respectively). Thus, it appears that patients with primary tumors in the rectum or the lung also have a higher risk for skeletal lesions.

\section{Complications in Patients with BM and Treatment Options}

In particular in the setting of a palliative treatment situation, quality of life remains one of the most important factors for patients with advanced NET. In our study, BM led to complications in nearly half of the patients, with pain being most common. While only 24 patients (28\%) reported pain at the time of initial diagnosis, another 14 patients (16.5\%) developed pain during follow-up. Bisphosphonates and denosumab nowadays are routinely used for other tumors, although a recent review by Porta-Sales et al. [23] did not find evidence, which supports an analgesic effect of either agent. Treatment with these compounds seems to delay the onset of bone pain, suggesting that is important to start treatment early, even when the patient is asymptomatic. In our study patients without symptoms had a superior OS in comparison to patients with pain or any other kind of symptom caused by BM ( $p=0.03)$. Moreover, patients who received a BMdirected treatment such as bisphosphonates or PRRT tended to have a slightly better median OS, even though the difference was not significant $(p=0.39)$.

Neuroendocrinology 2018;106:30-37 DOI: $10.1159 / 000457954$ 
Another valid option for the treatment of pain caused by $\mathrm{BM}$ is palliative radiation, which has demonstrated a positive effect on the quality of life [24]. Neurological complications such as compression of the spinal cord were only seen in 4 out of 85 patients, which is significantly less than observed in the study by Van Loon et al. [20]. However, in the study by Van Loon et al., the high number of neurological symptoms only occurred when the primary tumor was either a pheochromocytoma or paraganglioma, which were both not included in our study. Nevertheless, these complications have to be kept in mind by every physician taking care of patients with advanced NEN.

Apart from bisphosphonates and local therapies such as radiation when BM are diagnosed, NEN patients should be treated as recommended by the ENETS guidelines $[5,6,25]$, preferably using a systemic treatment such as PRRT, chemotherapy or targeted agents. While no systemic treatment has been demonstrated to be effective in terms of tumor response in patients with BM in prospective studies, some retrospective studies indicate that there is an advantage for PRRT-based therapies [26]. PRRT should therefore be considered when the tumor and the metastases are receptor positive.

\section{Prognostic Impact of BM}

Decisions in favor of one or the other treatment option are based on various properties of NEN. Grading, functionality, tumor burden, primary site and somatostatin receptor status nowadays are routinely taken into account [25]. Based on our data, the existence of BM should be included as a new factor on this list.

The retrospective analysis of all patients with BM treated at our ENETS center in the past 15 years clearly suggests that $\mathrm{BM}$ are a highly significant factor directly influencing the median OS in NEN patients. Irrespective of the primary site, grading or age of patients, BM have to be considered in the treatment of every individual NEN patient. Therefore, in our center, DOTATOC-PET-CT is routinely included in the diagnostic workup at the initial presentation and is usually repeated every 2 years to ensure the best individual evaluation and treatment of each patient.

\section{Limitations of our Study}

Despite the large number of patients treated at our center, this remains a retrospective analysis with all associated limitations. Ga-DOTATOC-based PET-CT, for instance, has only been introduced at our hospital in 2013. This may have led to 2 confounding circumstances. The overall rate of BM in our cohort may have been underestimated since
PET-CT was not available in a large number of patients that were not staged with ${ }^{68} \mathrm{Ga}$-DOTATOC-PET-CT. Additionally, the BM diagnosed before the introduction of PET-CT at our center may have been more advanced and therefore more often symptomatic. Otherwise, MRI, which is already highly sensitive to BM, is routinely used in our follow-up, while the less sensitive CT scans are more common in the US [19]. This difference in follow-up examinations may explain the slightly higher incidence of $\mathrm{BM}$ in our study, as well as fewer complications that may have been avoided due to an earlier detection. In this context, we also have to take into account that only in 1 of 85 patients BM was histologically confirmed (1.2\%), since our center strongly advocates using the site most easily accessible and least dangerous for the patient for biopsy to confirm stage IV. We can thus not exclude that we included some false-positive BM diagnoses due to granulomatous diseases or intraosseous hemangioma. However, it is unlikely that this had a major impact on our results.

Moreover, while our data suggest that BM have a negative impact on median OS, the groups compared in our study were not prospectively matched in regard to gender, age, differentiation of the tumor and other factors. For instance, since the extent of tumor load was not precisely measured in every patient, this might be another confounding variable, even though the general patient characteristics are quite similar and no obvious differences in tumor load were documented in the routine imaging reports and diagnoses. The number of patients with poorly differentiated tumors (G3) was slightly higher in the group with $\mathrm{BM}$, as was the median $\mathrm{Ki}-67$ value, while the percentage of NET originating from the small intestine was slightly lower (8.3\%). Based on the literature, both factors are known to have a negative impact on OS. Nevertheless, BM remained a significant prognostic parameter for OS in our multivariate analysis.

\section{Conclusion}

The presence of BM is of high prognostic and clinical relevance. Prospective studies are needed in order to analyze the significance of early detection due to improved imaging and whether diagnosis should influence our therapeutic decisions, in particular when BM are asymptomatic. Furthermore, drugs currently available, such as bisphosphonates and RANK inhibitors, have to be prospectively evaluated in patients with NEN and BM to study whether they can prevent pain and complications and improve OS.
36

Neuroendocrinology 2018;106:30-37 DOI: $10.1159 / 000457954$
Scharf/Petry/Daniel/Rinke/Gress 


\section{References}

1 Lawrence B, et al: The epidemiology of gastroenteropancreatic neuroendocrine tumors. Endocrinol Metab Clin North Am 2011;40: 1-18, vii.

2 Kloppel G: Classification and pathology of gastroenteropancreatic neuroendocrine neoplasms. Endocr Relat Cancer 2011;18(suppl 1):S1-S16.

3 Cives M, Strosberg J: An update on gastroenteropancreatic neuroendocrine tumors. Oncology (Williston Park) 2014;28:749-756, 758.

4 Halperin DM, Kulke MH, Yao JC: A tale of two tumors: treating pancreatic and extrapancreatic neuroendocrine tumors. Annu Rev Med 2015;66:1-16.

5 Pavel M, et al: ENETS Consensus Guidelines for the management of patients with liver and other distant metastases from neuroendocrine neoplasms of foregut, midgut, hindgut, and unknown primary. Neuroendocrinology 2012;95:157-176.

6 Kos-Kudla B, et al: ENETS consensus guidelines for the management of bone and lung metastases from neuroendocrine tumors. Neuroendocrinology 2010;91:341-350.

7 Pape UF, et al: Prognostic factors of long-term outcome in gastroenteropancreatic neuroendocrine tumours. Endocr Relat Cancer 2008; 15:1083-1097.

8 Garcia-Carbonero R, et al: Incidence, patterns of care and prognostic factors for outcome of gastroenteropancreatic neuroendocrine tumors (GEP-NETs): results from the National Cancer Registry of Spain (RGETNE). Ann Oncol 2010;21:1794-1803.

9 Lombard-Bohas C, et al: Thirteen-month registration of patients with gastroenteropancreatic endocrine tumours in France. Neuroendocrinology 2009;89:217-222.
10 Meijer WG, et al: Bone metastases in carcinoid tumors: clinical features, imaging characteristics, and markers of bone metabolism. J Nucl Med 2003;44:184-191.

11 Mignon M: Natural history of neuroendocrine enteropancreatic tumors. Digestion 2000;62(suppl 1):51-58.

12 Milone F, et al: Assessment and clinical implications of RANK/RANKL/OPG pathway as markers of bone tumor progression in patients with NET harboring bone metastases. Biomarkers 2013;18:121-125.

13 Janson ET, et al: Carcinoid tumors: analysis of prognostic factors and survival in 301 patients from a referral center. Ann Oncol 1997;8: 685-690.

14 Ross EM, Roberts WC: The carcinoid syndrome: comparison of 21 necropsy subjects with carcinoid heart disease to 15 necropsy subjects without carcinoid heart disease. Am J Med 1985;79:339-354.

15 Johnbeck CB, Knigge U, Kjaer A: PET tracers for somatostatin receptor imaging of neuroendocrine tumors: current status and review of the literature. Future Oncol 2014;10:22592277.

16 Caplin ME, et al: Pulmonary neuroendocrine (carcinoid) tumors: European Neuroendocrine Tumor Society expert consensus and recommendations for best practice for typical and atypical pulmonary carcinoids. Ann Oncol 2015;26:1604-1620.

17 Etchebehere EC, et al: 68Ga-DOTATATE PET/CT, 99mTc-HYNIC-octreotide SPECT/ $\mathrm{CT}$, and whole-body MR imaging in detection of neuroendocrine tumors: a prospective trial. J Nucl Med 2014;55:1598-1604.
18 Albanus DR, et al: Clinical value of (68)GaDOTATATE-PET/CT compared to standalone contrast enhanced CT for the detection of extra-hepatic metastases in patients with neuroendocrine tumours (NET). Eur J Radiol 2015;84:1866-1872.

19 Kavecansky J, et al: Bone metastases in wellto-moderately differentiated neuroendocrine tumors: a single institutional review from the Ohio State University Medical Center. Pancreas 2015;44:198-203.

20 Van Loon K, et al: Bone metastases and skeletal-related events from neuroendocrine tumors. Endocr Connect 2015;4:9-17.

21 Bhosale P, et al: Carcinoid tumours: predicting the location of the primary neoplasm based on the sites of metastases. Eur Radiol 2013;23:400-407.

22 Zuetenhorst JM, et al: Evaluation of (111)Inpentetreotide, (131)I-MIBG and bone scintigraphy in the detection and clinical management of bone metastases in carcinoid disease. Nucl Med Commun 2002;3:735-741.

23 Porta-Sales J, et al: Evidence on the analgesic role of bisphosphonates and denosumab in the treatment of pain due to bone metastases: a systematic review within the European Association for Palliative Care guidelines project. Palliat Med 2017;31:5-25.

24 McDonald R, et al: Quality of life after palliative radiotherapy in bone metastases: a literature review. J Bone Oncol 2015;4:24-31.

25 Pavel M, et al: ENETS consensus guidelines update for the management of distant metastatic disease of intestinal, pancreatic, bronchial neuroendocrine neoplasms (NEN) and NEN of unknown primary site. Neuroendocrinology 2016;103:172-185.

26 Sabet A, et al: Bone metastases in GEP-NET response and long-term outcome after PRRT from a follow-up analysis. Am J Nucl Med Mol Imaging 2013;3:437-445. 